Archives de sciences sociales des religions

112 | octobre-décembre 2000

Âme et corps : conceptions de la personne

\title{
HELL (Bertrand), Possession et chamanisme, les \\ maîtres du désordre
}

Paris, Flammarion, 1999, 392 p.

Erwan Dianteill

\section{OpenEdition}

\section{Journals}

Édition électronique

URL : http://journals.openedition.org/assr/20315

DOI : $10.4000 /$ assr. 20315

ISSN : $1777-5825$

\section{Éditeur}

Éditions de l'EHESS

Édition imprimée

Date de publication : 31 décembre 2000

Pagination : 98-100

ISBN : 2-222-96698-1

ISSN : 0335-5985

\section{Référence électronique}

Erwan Dianteill, « HELL (Bertrand), Possession et chamanisme, les maîtres du désordre », Archives de sciences sociales des religions [En ligne], 112 I octobre-décembre 2000, document 112.27, mis en ligne le 19 août 2009, consulté le 21 septembre 2020. URL : http://journals.openedition.org/assr/20315 ;

DOI : https://doi.org/10.4000/assr.20315

Ce document a été généré automatiquement le 21 septembre 2020

(c) Archives de sciences sociales des religions 


\section{HELL (Bertrand), Possession et chamanisme, les maîtres du désordre}

Paris, Flammarion, 1999, 392 p.

\section{Erwan Dianteill}

\section{RÉFÉRENCE}

HELL (Bertrand), Possession et chamanisme, les maitres du désordre, Paris, Flammarion, $1999,392 \mathrm{p}$.

1 Avec cet ouvrage, B.H. renouvelle profondément l'anthropologie du chamanisme et des phénomènes de possession et de transe. L'originalité de son approche, exposée dans une première partie, est double. D'abord, B.H. renoue avec la tradition d'une anthropologie générale prenant pour objet des faits culturels présents dans un grand nombre de sociétés. Spécialiste des Gnawa du Maroc, l'auteur ne se contente pas de faire l'ethnographie de ce groupe, il vise à "confronter les hypothèses issues de l'observation des pratiques marocaines à d'autres réalités ethnographiques et de les retravailler jusqu'à ce qu'elles touchent véritablement au noyau anthropologique sousjacent à l'ensemble du phénomène» (p. 16). Ensuite, il refuse la dichotomie établie entre chamanisme et possession. En effet, dans la perspective structuraliste, l'âme du chamane se libère de son corps pour faire un voyage au pays des esprits, alors que le corps du possédé est occupé provisoirement par une entité surnaturelle. Dans le premier cas, l'homme va chez les esprits, dans le second, c'est l'esprit qui visite les hommes. Or, en premier lieu, il apparait que cette dichotomie est beaucoup moins tranchée dans les faits : dans bien des cas, comme celui du culte de Maria Lionza au Venezuela par exemple, la possession s'accompagne de références à un voyage mystique. En second lieu, B.H. soutient qu'au-delà de l'opposition formelle, on a affaire à des "maitres du désordre ", dans le cas du chamane comme dans celui du possédé. En se focalisant sur le mode de contact avec les esprits, on perd de vue que chamanes et possédés jouent un rôle social équivalent, celui de répondre aux malheurs et aux 
infortunes qui affectent individus et communautés. C'est pourquoi B.H. propose de s'interroger sur les ressorts de l'efficacité symbolique propre au chamanisme et à la possession.

Elle repose d'abord sur une série de représentations concernant le monde des esprits. C'est l'objet de la deuxième partie de l'ouvrage. Les esprits sont des entités versatiles, insaisissables et surtout caractérisées par une profonde ambivalence : «les esprits qui protègent sont aussi ceux qui frappent» (p.130). Les esprits les plus dangereux, mais aussi les plus puissants, sont ceux qui « habitent» les espaces les plus éloignés de la sphère domestique. Ceux qui résident dans les friches et les forêts sont les plus sauvages, et en milieu urbain, les plus violents sont ceux qui rôdent dans les lieux publics, les carrefours et les ruelles obscures. Confrontés aux maux les plus rebelles, les individus consultent le chamane ou le possédé qui établit la source surnaturelle de l'infortune par la divination et accomplit les rites nécessaires à l'apaisement des esprits. Réunissant ces différents éléments, l'A. construit un tableau (p. 165) dans lequel les axes des esprits et du paysage sont mis en relation avec l'axe du malheur et celui des réparateurs de désordre. Les chamanes-possédés sont les alliés des esprits les plus sauvages, vivant dans les lieux les plus inhospitaliers et responsables des maux les plus graves. Ces spécialistes du désordre s'opposent aux prêtres et aux guérisseurs qui remédient aux troubles ordinaires produits par les esprits domestiques résidant dans les espaces les plus familiers.

3 À partir de ce modèle, B.H. montre dans une troisième partie que les maîtres du désordre occupent une situation très particulière dans l'espace social et symbolique. Ils se caractérisent toujours par l'étrangeté et la marginalité. Les Gnawa sont par exemple les descendants des esclaves noirs asservis par les Arabes et appartiennent aujourd'hui aux groupes les plus pauvres de la société marocaine, outre qu'ils sont victimes de discriminations "raciales ». En fait, on attribue très souvent aux chamanes et aux possédés les caractéristiques des esprits qu'ils «fréquentent». Ils sont considérés comme des individus instables, violents, insaisissables et dangereux. Les rituels thérapeutiques font écho à ces caractéristiques. Cris, tremblements, gesticulations, manifestations d'insensibilité à la douleur, insultes ou propos grivois s'opposent au comportement et au langage bienséants dans la vie quotidienne. De plus, les maitres du désordre transgressent systématiquement nombre de normes collectives, en matière sexuelle en particulier. L'identité sexuelle du chamane-possédé est fréquemment indéterminée, car, entre deux mondes, il est aussi entre deux sexes. La furie, l'altérité, la transgression et l'ambiguïté, telles sont les « marques du désordre ».

Dans une dernière partie, B.H. propose une "philosophie du désordre " qui vise à mettre au jour les ressorts de l'efficacité symbolique de la possession et du chamanisme. Celle-ci repose sur deux piliers : l'idée d'une alliance entre le chamanepossédé et les esprits d'un côté, et de l'autre sur le pragmatisme et l'adaptabilité des pratiques thérapeutiques. Le premier contact entre homme et esprit est presque toujours douloureux pour le premier: l'esprit est un fauteur de troubles. Mais si la personne perturbée parvient à dépasser ce premier stade par des sacrifices, cette relation se modifie, elle prend la forme d'une alliance, parfois celle d'un mariage explicite, et même, dans le cas du vaudou haïtien, d'un contrat écrit de mariage en bonne et due forme. Ainsi, se libérer du malheur ne passe pas du tout par l'exorcisme et l'expulsion définitive des esprits du monde des hommes. Tout au contraire, le retour à l'équilibre est fait de négociation et de marchandage avec un esprit dont on est jamais 
"débarrassé »: le pacte de protection doit toujours être renouvelé par des offrandes. C'est pourquoi possession et chamanisme sont réfractaires au dogmatisme, à la fixation d'une orthodoxie ou d'une orthopraxie. Rétifs à la codification, ils sont gouvernés au contraire par un souci d'adaptation à toutes les situations de troubles individuels et collectifs. Enfin, ils reposent sur une conception du monde qui privilégie l'alternance de l'ordre et du désordre plutôt que l'illusoire élimination des aléas de l'existence humaine, comme c'est le cas en Occident.

Quelques points soulevés par B.H. mériteraient d'être particulièrement approfondis. En premier lieu, le concept de "surnature » utilisé par B.H. pour désigner le monde des esprits est problématique. Ces derniers sont-ils considérés par les chamanes-possédés comme des êtres "surnaturels ", c'est-à-dire transcendants par rapport au monde des hommes? On peut en douter puisqu'ils interviennent si fréquemment dans l'existence humaine, et que les plus dangereux sont précisément ceux qui sont les plus sauvages, c'est-à-dire les plus liés à la «nature », opposée cette fois à la «culture » au sens de C. Lévi-Strauss. Il faudrait donc examiner sur le plan théorique toutes les valences de la triade conceptuelle « surnature/nature/culture ». En second lieu, si le mariage est bien une figure clef de la relation entre homme et esprit, est-elle toujours la seule valence active dans la relation aux esprits ? B.H. est conscient qu'il existe un autre registre de la parenté utilisé par les possédés et les chamanes: celui de la filiation. Il faudrait examiner, dans des cas précis, la dynamique de combinaison de l'alliance et de la filiation que permet la possession rituelle. On sait que dans la santeria cubaine ou le vaudou haïtien, par exemple, l'articulation du mariage mystique et de la filiation divine permet l'engendrement d'un filleul par un parrain et constitue ainsi une lignée de croyants. Enfin, l'opposition entre religion dogmatique, administrée par des prêtres garants de l'ordre social, et possession-chamanisme, animés par des individus marginaux et transgressifs qui manipulent les forces du désordre, n'est-elle pas trop tranchée? On sait par exemple que la possession par l'Esprit Saint se déroule dans le cadre institutionnel des Églises pentecôtistes, ou bien qu'il existe des courants charismatiques parfaitement intégrés à l'Eglise catholique. Dans ces deux cas, pasteurs et prêtres «administrent le sacré ». Même au sein de "culte de possession » comme la santeria, la coopération est nécessaire entre possédés, souvent des femmes et des hommes homosexuels, et les spécialistes de la divination, les babalaos, toujours des hommes hétérosexuels qui ne doivent en aucun cas être sujets à la transe. En effet, ce sont ces derniers qui sont chargés des sacrifices nécessaires à l'initiation des premiers. De plus, si la coopération entre prêtres et chamanes-possédés n'est pas exclue, l'institutionnalisation du chamanisme n'est pas non plus impossible. N'est-ce pas ce que l'on observe au Guatemala avec la naissance d'une Église Maya?

6 L'ouvrage de B.H. est donc extrêmement stimulant et ouvre de nombreuses pistes de recherches, autant sur la question des techniques de l'efficacité symbolique, des rapports entre magie et religion, du rapport cognitif aux entités spirituelles que sur la question plus générale de la gestion symbolique du désordre dans l'existence humaine. 